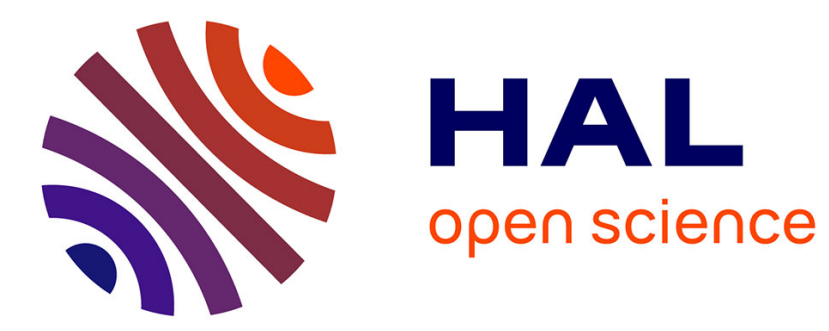

\title{
An approximate yield criterion for porous single crystals
} Joseph Paux, Léo Morin, Renald Brenner, Djimédo Kondo

\section{To cite this version:}

Joseph Paux, Léo Morin, Renald Brenner, Djimédo Kondo. An approximate yield criterion for porous single crystals. 2014. hal-01062662

\section{HAL Id: hal-01062662 https://hal.science/hal-01062662}

Preprint submitted on 10 Sep 2014

HAL is a multi-disciplinary open access archive for the deposit and dissemination of scientific research documents, whether they are published or not. The documents may come from teaching and research institutions in France or abroad, or from public or private research centers.
L'archive ouverte pluridisciplinaire HAL, est destinée au dépôt et à la diffusion de documents scientifiques de niveau recherche, publiés ou non, émanant des établissements d'enseignement et de recherche français ou étrangers, des laboratoires publics ou privés. 


\title{
An approximate yield criterion for porous single crystals
}

\author{
J. Paux, L. Morin, R. Brenner*, D. Kondo \\ Sorbonne Universités, UPMC Univ. Paris 06, CNRS, UMR 7190, \\ Institut Jean Le Rond d'Alembert, F-75005, Paris, France
}

\begin{abstract}
This study is devoted to the derivation of an approximate yield function for voided single crystals deforming by crystallographic slip. By making use of a regularized form of the Schmid law and solving approximately the hydrostatic case with a limit-analysis calculation, a Gurson-type yield criterion is proposed. It is obtained by a heuristic extension of existing limit-analysis results for a matrix obeying a quadratic Hill-type criterion. The proposed yield function is successfully compared with numerical results from the literature for face-centered cubic crystals.
\end{abstract}

Keywords: single crystal, porosity, plasticity, limit-analysis

\section{Introduction}

The ductile fracture of crystalline materials is a classical subject in mechanics of materials which still presents important challenges. It is driven by the process of growth and coalescence of voids and thus calls for constitutive models of the plastic response of porous crystalline materials. In this context, the consideration of the anisotropy, related to the single crystal response and the morphological and crystallographic textures of polycrystals, is a key issue.

Important progresses have been made in the description of the plastic behaviour of polycrystals containing cavities with mainly two different approaches. On the one hand, a large class of models has been derived in the framework of limit-analysis, following the pioneering model of Gurson [1]. This model has been obtained from homogenization, using a limit-analysis of a hollow sphere subjected on its outer surface to homogeneous strain rate conditions [2, 3]. Many investigations have been devoted to extend this model: (i) to include void shape effects by considering first spheroidal voids $[4,5,6]$ and then general ellipsoidal voids $[7,8]$ and (ii) to include crystallographic texture effects, by considering a matrix material obeying an orthotropic Hill yield criterion $[9,10,11]$. The reader is referred to $[12,13]$ for a critical assessment of these criteria. On the other hand, variational nonlinear homogenization approaches $[14,15,16,17]$ have been applied to textured viscoplastic polycrystals with intergranular cavities [18] and perfectly plastic voided polycrystals subjected to a hydrostatic loading [19], among others. Besides, full-field numerical simulations have been performed to study void growth and coalescence within polycrystalline aggregates [18, 20].

As compared to the studies on polycrystals, relatively few works exist on the constitutive response of plastic single crystals containing voids. However, the importance of the crystalline anisotropy to describe the stress state surrouding intragranular voids has been clearly evidenced analytically, experimentally and numerically $[21,22,23,24,25]$. It is worth citing the study based on an improved variational homogenization method [17] which has been undertaken to derive the effective flow stress

\footnotetext{
*Corresponding author

Email address: renald.brenner@upmc.fr (R. Brenner)
} 
of a model 2D single crystal containing cylindrical voids and subjected to antiplane shear [26]. Also, a yield function for 3D porous single crystals has been recently derived by using the variational homogenization method proposed in [27] and ad hoc modifications inspired by limit-analysis results [28]. By making use of the Schmid law, the authors obtain a multi-criterion yield function.

The objective of the present study is to derive a Gurson-type yield function for porous single crystals. Due to the widely spread finite-element (FE) implementations of the Gurson model, it would present an obvious interest to consider the case of single crystals deforming by crystallographic slip. With this aim in view, use is made of a regularization of the Schmid law [29, 30]. This key ingredient allows us to obtain a single yield function defining the plastic strength domain of voided single crystals. This feature is a definite advantage with respect to previous proposals. The proposed criterion is assessed by comparison with results from unit-cell FE computations performed in [28] for Face-Centered Cubic (FCC) single crystals with various orientations.

\section{Plastic strength domain of the single crystal}

We consider that the constitutive single crystal can undergo plastic deformation only by dislocation glide on specific crystalline planes depending on the crystal structure. A slip system $k$ is geometrically characterized by the Schmid tensor $\boldsymbol{\mu}_{k}$

$$
\boldsymbol{\mu}_{k}=\frac{1}{2}\left(\mathbf{m}_{k} \otimes \mathbf{n}_{k}+\mathbf{n}_{k} \otimes \mathbf{m}_{k}\right)
$$

with $\mathbf{n}_{k}$ the unit normal to the slip plane and $\mathbf{m}_{k}$ the unit slip direction.

\subsection{Schmid yield criterion}

According to the Schmid law [31], plastic deformation occurs on a slip system $k$, at a given point $\mathbf{x}$, if the absolute value of the resolved shear stress $\tau_{k}(\mathbf{x})$, defined by

$$
\tau_{k}(\mathbf{x})=\boldsymbol{\mu}_{k}: \boldsymbol{\sigma}(\mathbf{x}) \text { with } \boldsymbol{\sigma} \text { the Cauchy stress tensor, }
$$

reaches a threshold value $\tau_{k}^{0}$. Consequently, the plastic strength domain $C$ of a single crystal with $K$ slip systems is defined by a multi-criterion

$$
C=\left\{\boldsymbol{\sigma} \text { such that } f_{k}(\boldsymbol{\sigma}) \leq 0, \forall k=1, \ldots, K\right\}
$$

with $f_{k}(\boldsymbol{\sigma})=\left|\boldsymbol{\mu}_{k}: \boldsymbol{\sigma}\right|-\tau_{k}^{0}$. The yield function of the single crystal is thus non regular and reads

$$
f(\boldsymbol{\sigma})=\sup _{k=1, \ldots, K} f_{k}(\boldsymbol{\sigma}) \leq 0 .
$$

Following the generalized normality rule, the plastic strain rate tensor $\mathbf{d}$ can be written as

$$
\mathbf{d}=\sum_{k=1}^{K} \dot{\gamma}_{k} \frac{\partial f_{k}}{\partial \boldsymbol{\sigma}}(\boldsymbol{\sigma}), \quad \dot{\gamma}_{k} \geq 0, \quad \dot{\gamma}_{k}=0 \quad \text { if } \quad f_{k}(\boldsymbol{\sigma})<0 .
$$

The plastic multipliers $\dot{\gamma}_{k}$ are the slip rates on each slip system. 


\subsection{Regularized Schmid law}

For practical purposes, it can be convenient to remove the yield surface singularities resulting from the multi-criterion (3). This regularization of the Schmid law consists in approximating the multicriterion yield function $f(\boldsymbol{\sigma})$ (4) by a differentiable and strictly convex single yield function $f_{\text {reg }}(\boldsymbol{\sigma})$. This has been initially proposed in $[29,30]$ with the following form

$$
f_{\text {reg }}(\boldsymbol{\sigma})=\left(\sum_{k=1}^{K}\left(\frac{\left|\boldsymbol{\mu}_{k}: \boldsymbol{\sigma}\right|}{\tau_{k}^{0}}\right)^{n}\right)^{1 / n}-1 \leq 0, \quad n \geq 2 .
$$

It can be noted for later reference that a similar approximation had been previously proposed in [32] for the particular case $n=2$ (i.e quadratic yield criterion). As $n \rightarrow+\infty$, the regularized function $f_{r e g}(\boldsymbol{\sigma})$ tends to $f(\boldsymbol{\sigma})$. With the normality rule, the plastic strain rate tensor $\mathbf{d}$ reads

$$
\mathbf{d}=\dot{\lambda} \frac{\partial f_{r e g}}{\partial \boldsymbol{\sigma}}(\boldsymbol{\sigma}), \quad \dot{\lambda} \geq 0, \quad \dot{\lambda}=0 \quad \text { if } \quad f(\boldsymbol{\sigma})<0 .
$$

In contradistinction to the non regular Schmid criterion, all slip systems are active when the yield surface is reached $\left(f_{\text {reg }}(\boldsymbol{\sigma})=0\right)$. By noting that

$$
\frac{\partial f_{r e g}}{\partial \boldsymbol{\sigma}}=\sum_{k=1}^{K} \frac{\partial f_{r e g}}{\partial \tau_{k}} \frac{\partial \tau_{k}}{\partial \boldsymbol{\sigma}}
$$

the slip rates on each slip system $\dot{\gamma}_{k}$ are obtained as functions of the unique plastic multiplier $\dot{\lambda}$

$$
\dot{\gamma}_{k}=\dot{\lambda} \frac{\partial f_{r e g}}{\partial \tau_{k}}=\frac{\dot{\lambda}}{\tau_{0}^{k}}\left(\frac{\left|\tau_{k}\right|}{\tau_{k}^{0}}\right)^{n-1} \operatorname{sgn}\left(\tau_{k}\right)
$$

It can be remarked that this expression is similar to the one corresponding to crystalline viscoplasticity with a power-law flow rule. However, the $n$ exponent has completely different meanings. In the viscoplastic context, it corresponds to the coefficient of stress sensitivity and a rate-independent behaviour is asymptotically obtained as $n \rightarrow+\infty$. In the present case, the $n$ exponent is only related to the geometrical approximation of the yield surface and the plastic behaviour is rate-independent whatever the $n$ value. Regularized yield functions, with a scaling ensuring a match with vertices of the Schmid yield surface for all $n$ values $[32,33]$, are shown on Figure 1.

\section{Approximation of the yield function of voided single crystals}

\subsection{Description of the single hollow sphere}

We aim to derive an approximation of the yield surface of rigid-perfectly plastic single crystals containing spherical voids by taking advantage of the regularized Schmid law (6). To this end, it is proposed to follow a kinematical limit-analysis approach on a hollow sphere. Following [1,9] the cell considered for the porous medium is a sphere $\Omega$ containing a confocal spherical void $\omega$ (Figure 2). The interior radius is denoted $a$ and the exterior one $b$. The porosity is defined by $f=\operatorname{vol}(\omega) / \operatorname{vol}(\Omega)$ with $\operatorname{vol}(\Omega)=(4 \pi / 3) b^{3}$ the total volume and $\operatorname{vol}(\omega)=(4 \pi / 3) a^{3}$ the cavity volume. The outer surface of the sphere is $\partial \Omega$ and the surface of the pore is $\partial \omega$. 


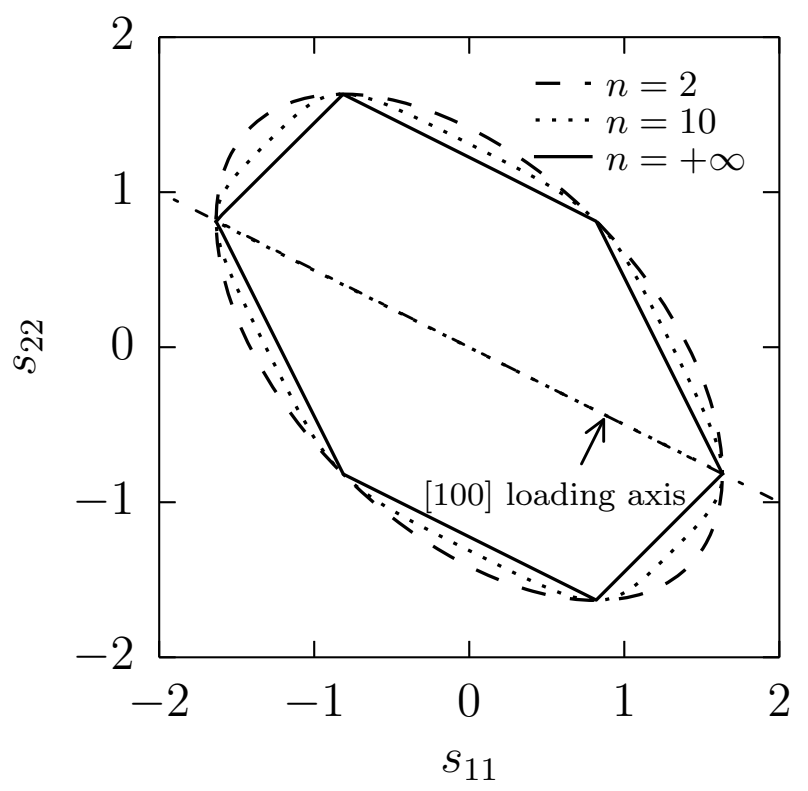

Figure 1: Section of regularized Schmid yield functions and the Bishop-Hill polyhedron [34] in the deviatoric stress plane $\left(s_{11}, s_{22}\right)$. The axial loading corresponding to the [100] crystalline direction is indicated.

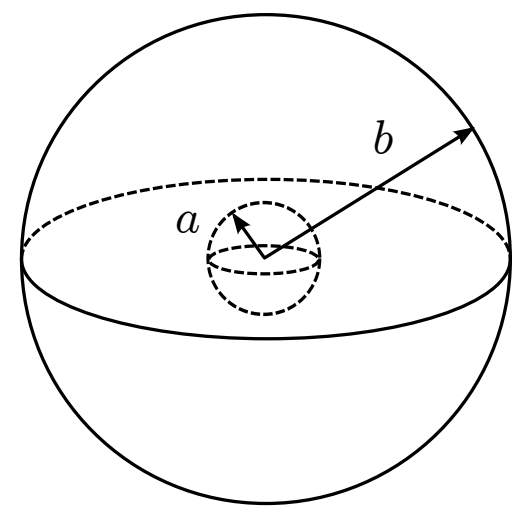

Figure 2: Cell considered for the porous material in the kinematical limit-analysis approach.

\subsection{Kinematical limit-analysis approach}

It is assumed that the cell is subjected on its outer boundary to homogeneous strain rate conditions, that is

$$
\mathbf{v}(\mathbf{x})=\mathbf{D} \cdot \mathbf{x}, \quad \forall \mathbf{x} \in \partial \Omega
$$

with $\mathbf{v}$ the velocity field and $\mathbf{D}$ the macroscopic strain rate tensor. The single crystal presents a convex plastic strength domain $C$ and, according to the principle of maximum plastic power, the local plastic dissipation function (a.k.a support function) is defined by

$$
\pi(\mathbf{d})=\sup _{\boldsymbol{\sigma}^{*} \in C} \boldsymbol{\sigma}^{*}: \mathbf{d}, \quad \forall x \in \Omega-\omega
$$


with $\mathbf{d}$ the strain rate field (symmetric part of the gradient of the velocity field $\mathbf{v}$ ). For the porous material, the effective plastic dissipation function reads [35]

$$
\Pi(\mathbf{D})=\inf _{\mathbf{v} \in \mathcal{K}(\mathbf{D})}\langle\pi\rangle_{\Omega}=(1-f) \inf _{\mathbf{v} \in \mathcal{K}(\mathbf{D})}\langle\pi\rangle_{\Omega-\omega}
$$

with $\mathcal{K}$ the set of kinematically admissible velocity field

$$
\mathcal{K}(\mathbf{D})=\left\{\mathbf{v} \text { such that }\langle\mathbf{d}\rangle_{\Omega}=\mathbf{D}, \operatorname{tr}(\mathbf{d}(\mathbf{x}))=\mathbf{0}, \forall \mathbf{x} \in \Omega-\omega\right\} .
$$

The upper-bound of the effective strength domain $\widetilde{C}$ of the porous single crystal is defined as

$$
\widetilde{C}=\{\boldsymbol{\Sigma} \text { such that } \boldsymbol{\Sigma}: \mathbf{D} \leq \Pi(\mathbf{D}), \forall \mathbf{D}, \operatorname{div}(\boldsymbol{\sigma})=\mathbf{0}, \boldsymbol{\sigma} \in C, \forall \mathbf{x} \in \Omega-\omega\}
$$

with $\boldsymbol{\Sigma}$ the macroscopic stress tensor. The corresponding macroscopic yield surface, defined as the boundary of the convex strength domain $\widetilde{C}$, is thus given by

$$
\boldsymbol{\Sigma}=\frac{\partial \Pi}{\partial \mathbf{D}}(\mathbf{D})
$$

Its derivation thus requires the determination of the support function $\pi(\mathbf{d})$ and to minimize its integral over the sphere volume with respect to the velocity field (12).

\subsection{Gurson-type yield criterion using regularized Schmid law}

For sake of simplicity, it is considered in the sequel that the critical shear stresses do not depend on the slip system, that is $\tau_{k}^{0}=\tau_{0}, \forall k$. In this particular case, the regularized yield function (6) is expressible as

$$
f_{\text {reg }}(\boldsymbol{\sigma})=\left[\sum_{k=1}^{K}\left|\tau_{k}\right|^{n}\right]^{1 / n}-\tau_{0} \leq 0, \quad n \geq 2,
$$

and the plastic dissipation of the single crystal reads

$$
\boldsymbol{\pi}(\mathbf{d})=\sup _{\boldsymbol{\sigma}^{*}, f_{r e g}\left(\boldsymbol{\sigma}^{*}\right) \leq 0} \boldsymbol{\sigma}^{*}: \mathbf{d}=\sup _{\boldsymbol{\sigma}^{*}, f_{r e g}\left(\boldsymbol{\sigma}^{*}\right) \leq 0} \dot{\lambda} \frac{\partial f_{r e g}}{\partial \boldsymbol{\sigma}^{*}}: \boldsymbol{\sigma}^{*}=\dot{\lambda} \tau_{0}
$$

with the plastic strain rate given by the normality rule (7)

$$
\mathbf{d}=\frac{\dot{\lambda}}{\left(\tau_{0}\right)^{n-1}} \sum_{k}\left|\boldsymbol{\mu}_{k}: \boldsymbol{\sigma}\right|^{n-1} \boldsymbol{\mu}_{k} \operatorname{sgn}\left(\tau_{k}\right) .
$$

By adopting a quadratic approximation for the regularized criterion (i.e $n=2$ ), we get a Hill-type anisotropic material, for which

$$
\mathbf{d}=\frac{\dot{\lambda}}{\tau_{0}} \mathbf{M}: \boldsymbol{\sigma} \quad \text { with } \quad \mathbf{M}=\sum_{k=1}^{K} \boldsymbol{\mu}_{k} \otimes \boldsymbol{\mu}_{k}
$$

which leads to the expression of the plastic multiplier $\dot{\lambda}$

$$
\dot{\lambda}=\sqrt{\mathbf{d}: \mathbf{H}: \mathbf{d}} .
$$

$\mathbf{H}$ is the inverse of the tensor $\mathbf{M}$ in the deviatoric space, that is $\mathbf{H}: \mathbf{M}=\mathbf{M}: \mathbf{H}=\mathbf{K}$ with $\mathbf{K}$ the isotropic deviatoric projector. Thence, the support function of the single crystal is given by

$$
\boldsymbol{\pi}(\mathbf{d})=\left\{\begin{array}{ccc}
\tau_{0} \sqrt{\mathbf{d}: \mathbf{H}: \mathbf{d}} & \text { if } & \operatorname{tr}(\mathbf{d})=0 \\
+\infty & \text { if } & \operatorname{tr}(\mathbf{d}) \neq 0
\end{array}\right.
$$


For single crystals presenting orthotropic or higher symmetry, the problem at hand defined by (12) is therefore the one considered in $[9,10]$ for a porous material constituted of a plastic matrix obeying the Hill yield criterion [36].

Hereafter, our analysis is restricted to the case of single crystals with cubic symmetry. The anisotropic deviatoric tensor $\mathbf{M}$ can thus be written

$$
\mathbf{M}=\alpha^{\prime} \mathbf{K}^{\prime}+\alpha^{\prime \prime} \mathbf{K}^{\prime \prime}
$$

with $\mathbf{K}^{\prime}$ and $\mathbf{K}^{\prime \prime}$ the cubic deviatoric projectors which verify $\mathbf{K}^{\prime}+\mathbf{K}^{\prime \prime}=\mathbf{K}[37]$. Since $\mathbf{K}^{\prime}$ and $\mathbf{K}^{\prime \prime}$ are orthogonal and idempotent, the inverse tensor $\mathbf{H}$ simply reads

$$
\mathbf{H}=\frac{1}{\alpha^{\prime}} \mathbf{K}^{\prime}+\frac{1}{\alpha^{\prime \prime}} \mathbf{K}^{\prime \prime}
$$

In the case of FCC crystals with twelve octahedral slip systems $\{111\}<\overline{1} 10>$, we have

$$
\mathbf{M}=\sum_{k=1}^{12} \boldsymbol{\mu}_{k} \otimes \boldsymbol{\mu}_{k}=2 \mathbf{K}^{\prime}+\frac{2}{3} \mathbf{K}^{\prime \prime}
$$

However, this approximation provides a poor approximation of the Bishop-Hill polyhedron [34]. It can be improved by minimizing the distance between the quadratic approximation and vertices of the Bishop-Hill polyhedron. This alternative approach, which has been developed in [32] for the study of the development of crystallographic texture of polycrystals, leads to

$$
\mathbf{M}=\frac{1}{4} \mathbf{K}^{\prime}+\frac{1}{9} \mathbf{K}^{\prime \prime}
$$

A section of the corresponding yield surface is shown in Figure 1. The definition (25) is adopted in the following.

Since the regularized criterion adopted to describe the matrix is a particular case of an orthotropic Hill criterion, we can make direct use of previous results from the limit-analysis of a hollow sphere made of an anisotropic Hill matrix $[9,10]$. The following quadratic criterion is thus obtained for single crystals with cubic symmetry containing spherical voids (Appendix A)

$$
\left(\frac{\sqrt{\boldsymbol{\Sigma}: \mathbf{M}: \mathbf{\Sigma}}}{\tau_{0}}\right)^{2}+2 f \cosh \left(\kappa \frac{\Sigma_{m}}{\tau_{0}}\right)-1-f^{2}=0
$$

with $\kappa$ an anisotropy coefficient defined by (A.6). For $n>2$, the explicit expression for the local plastic dissipation is not known yet. To address this case, we thus propose in the sequel a heuristic extension of the quadratic criterion.

\subsection{Improvements of the quadratic criterion}

The quadratic criterion (26) is likely to suffer from the approximate description of the Bishop-Hill polyhedron due to the quadratic regularization. In order to enhance this yield criterion, two distinct modifications are proposed.

Improvement for deviatoric loadings. In the special case $f=0$, the macroscopic criterion (26) reduces to

$$
\boldsymbol{\Sigma}: \mathbf{M}: \boldsymbol{\Sigma}-\tau_{0}^{2}=0 .
$$

It is clear from equation (27) that the sound material obeying Schmid criterion is not recovered in general, due to the choice $n=2$ in the regularization; however in some particular cases (e.g. the [100] 
crystalline direction), the regularized criterion coincides by construction with the Schmid criterion. This discrepancy (see Figure 1) will impact the porous material, at least for deviatoric loadings when the term in the hyperbolic cosine vanishes. In order to recover the sound material, it is proposed to substitute to the quadratic form $\boldsymbol{\Sigma}: \mathbf{M}: \boldsymbol{\Sigma}$ the following quantity

$$
\mathrm{Q}(\boldsymbol{\Sigma})=\left(\sum_{k=1}^{K}\left|\boldsymbol{\mu}_{k}: \boldsymbol{\Sigma}\right|^{n}\right)^{2 / n}
$$

where $n$ is taken large enough to ensure coincidence between the regularized and the Schmid criteria.

Improvement for hydrostatic loadings. The anisotropy parameter $\kappa$ (A.6), which affects the mean stress $\Sigma_{m}$, corresponds to a quadratic yield criterion. In the sequel, we consider its determination in the case of a face-centered cubic crystalline matrix obeying the Schmid law (multi-criterion yield function). The following strain rate field is adopted [1]

$$
\mathbf{d}=\frac{b^{3}}{r^{3}} D_{m} \mathbf{G} \quad \text { with } \quad \mathbf{G}=-2 \mathbf{e}_{r} \otimes \mathbf{e}_{r}+\mathbf{e}_{\theta} \otimes \mathbf{e}_{\theta}+\mathbf{e}_{\varphi} \otimes \mathbf{e}_{\varphi}
$$

where $\left(\mathbf{e}_{r}, \mathbf{e}_{\theta}, \mathbf{e}_{\varphi}\right)$ is the local orthonormal basis for spherical symmetry. To determine the plastic dissipation $\pi(\mathbf{d})$, we can take advantage of the fact that the Schmid yield surface is a convex polyhedron in the deviatoric stress space. As a consequence, the stress tensor which maximizes the plastic dissipation necessarily corresponds to a vertex of the polyhedron, that is

$$
\pi(\mathbf{d})=\sup _{\boldsymbol{\sigma}^{*} \in C} \boldsymbol{\sigma}^{*}: \mathbf{d}=\sup _{\boldsymbol{\sigma}^{*} \in V(C)} \boldsymbol{\sigma}^{*}: \mathbf{d}, \quad \forall x \in \Omega-\omega
$$

with $C$ the plastic strength domain of the single crystal $(3)$ and $V(C)$ the set of vertices of its boundary. The macroscopic plastic dissipation

$$
\Pi(\mathbf{D})=\frac{1}{\operatorname{vol}(\Omega)} \int_{\Omega} \pi(\mathbf{d}) \mathrm{d} \Omega=\frac{1}{\operatorname{vol}(\Omega)} \int_{r=a}^{b} \int_{\theta=0}^{2 \pi} \int_{\varphi=0}^{\pi} \pi(\mathbf{d}) r^{2} \sin \varphi \mathrm{d} \varphi \mathrm{d} \theta \mathrm{d} r
$$

then reads, by introducing (29) and (30),

$$
\Pi(\mathbf{D})=\frac{-D_{m} \ln f}{4 \pi} \int_{\theta=0}^{2 \pi} \int_{\varphi=0}^{\pi} \sup _{\boldsymbol{\sigma}^{*} \in V(C)} \boldsymbol{\sigma}^{*}: \mathbf{G} \sin \varphi \mathrm{d} \varphi \mathrm{d} \theta .
$$

By considering the set of vertices of the Bishop-Hill polyhedron, the double integral has been evaluated numerically

$$
I_{\pi}=\int_{\theta=0}^{2 \pi} \int_{\varphi=0}^{\pi} \sup _{\boldsymbol{\sigma}^{*} \in V(C)} \boldsymbol{\sigma}^{*}: \mathbf{G} \sin \varphi \mathrm{d} \varphi \mathrm{d} \theta \approx 74.56 \tau_{0} .
$$

The yield stress tensor for a hydrostatic loading reads

$$
\boldsymbol{\Sigma}=\frac{\partial \Pi}{\partial \mathbf{D}}(\mathbf{D})=\frac{-I_{\pi} \ln f}{12 \pi} \mathbf{i}
$$

with $\mathbf{i}$ the second-order identity tensor. Besides, the form of the yield criterion (26) implies

$$
\Sigma_{m}=\frac{-\tau_{0} \ln f}{\kappa^{\prime}} .
$$

The sought parameter $\kappa^{\prime}$ thus reads

$$
\kappa^{\prime}=\frac{12 \pi \tau_{0}}{I_{\pi}} \approx 0.506
$$




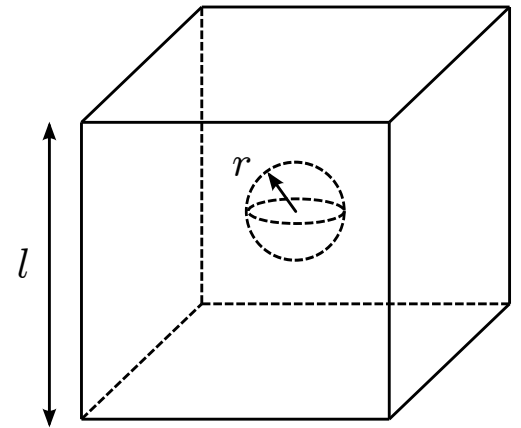

Figure 3: Unit-cell considered for the porous material in the numerical simulations of [28].

Interestingly, the value we derive semi-analytically is close to the one which has been obtained by parameter fitting in [28]. The value reported by Han et al. is $\kappa^{\prime}=1.325 \sqrt{(3 / 20)} \approx 0.513$. By taking account of the proposed improvements for purely deviatoric and purely hydrostatic loadings, the generalization of the yield criterion (26) is

$$
\left(\frac{\left(\sum_{k=1}^{K}\left|\boldsymbol{\mu}_{k}: \boldsymbol{\Sigma}\right|^{n}\right)^{1 / n}}{\tau_{0}}\right)^{2}+2 f \cosh \left(\kappa^{\prime} \frac{\Sigma_{m}}{\tau_{0}}\right)-1-f^{2}=0 .
$$

The evaluation of its relevance is considered hereafter.

\section{Numerical assessment of the proposed criteria for FCC crystals}

\subsection{Generalities}

In order to assess the criteria previously derived, numerical FE results on unit-cells composed of FCC crystals obeying the Schmid law and containing a single void [28] will be used. The unit-cell used in these numerical calculations is a cube of side $l$ containing a spherical void of radius $r$ (Figure $3)$. In the macroscopic reference frame $\left(\mathbf{e}_{1}, \mathbf{e}_{2}, \mathbf{e}_{3}\right)$, the overall stress tensor reads

$$
\boldsymbol{\Sigma}=\Sigma_{1}\left(\mathbf{e}_{1} \otimes \mathbf{e}_{1}+\eta_{2} \mathbf{e}_{2} \otimes \mathbf{e}_{2}+\eta_{3} \mathbf{e}_{3} \otimes \mathbf{e}_{3}\right)
$$

and various orientations of the single crystal are considered (see Appendix $\mathrm{C}$ for the definition of the orientation matrix).

Since the geometry considered in these numerical simulations differs from the one considered theoretically, a consistent definition of the porosity has to be adopted [38, 39]. Indeed, the cubic unit-cell loses its carrying capacity when the spherical void reaches the exterior boundary of the cube, that is for $f=\pi / 6 \simeq 0.52$; on the other hand, the spherical cell loses its carrying capacity for $f=1$. Following [38], the porosity $f$ considered in the model is replaced by $q f, q$ being a heuristic coefficient which plays a role of adjustment of the porosity (the numerical value of this coefficient will be discussed in the following). The general form of the proposed criterion finally reads

$$
\left(\frac{\left(\sum_{k=1}^{K}\left|\boldsymbol{\mu}_{k}: \boldsymbol{\Sigma}\right|^{n}\right)^{1 / n}}{\tau_{0}}\right)^{2}+2 q f \cosh \left(\kappa^{\prime} \frac{\Sigma_{m}}{\tau_{0}}\right)-1-(q f)^{2}=0 .
$$

It has to be remarked that this criterion shares similarities with the yield function proposed in [28] by using the variational homogenization method (Appendix B). 


\subsection{Shortcomings of the quadratic criterion}

To confirm the foreseen shortcomings of the criterion (26), axisymmetric loadings have been considered, that is $\eta_{2}=\eta_{3}=\eta$ with $\eta$ ranging from -0.5 (purely deviatoric loading) to 1 (purely hydrostatic loading). Illustrative results are presented in Figure 4 for a porosity of $1 \%$. To start with, it is observed that the original criterion (26) does not agree with the unit-cell FE computations whatever the crystalline orientation (comparisons have been made for numerous orientations which are not reported here for conciseness). A clear improvement is obtained with a phenomenological extension of the criterion (i.e $f$ replaced by $q f$ ) by setting $q=2.2$. A correct description of the yield stress is obtained over the whole range of stress triaxiality when the axisymmetric loading direction is aligned with the [100] crystalline direction. For this crystalline orientation, it is pointed out that the quadratic approximation for the bulk single crystal coincides, by construction, with the Schmid criterion (Figures 1 and 4a). It has been observed that a good match is obtained for the yield stress of the porous material when the axisymmetric loading direction corresponds to a vertex of the Bishop-Hill polyhedron of the single crystal. By contrast, a poor description is obtained for crystalline orientations which do not have this property (Figure 4b). It can be thus concluded that except for some specific orientations, the criterion (26), even with an enhanced porosity $q f$, does not provide a correct description of the yield surface of porous FCC single crystals.

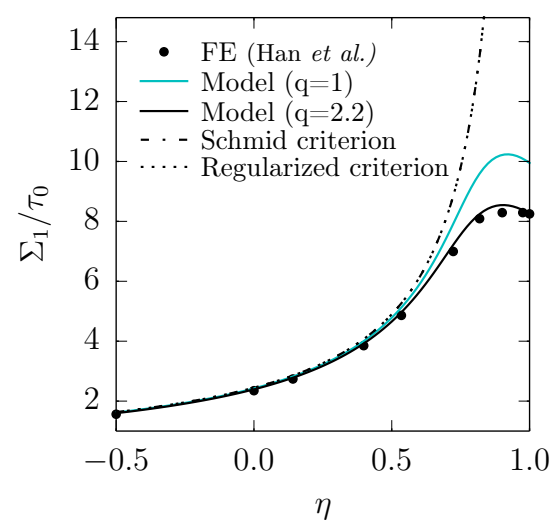

(a) [100] loading direction

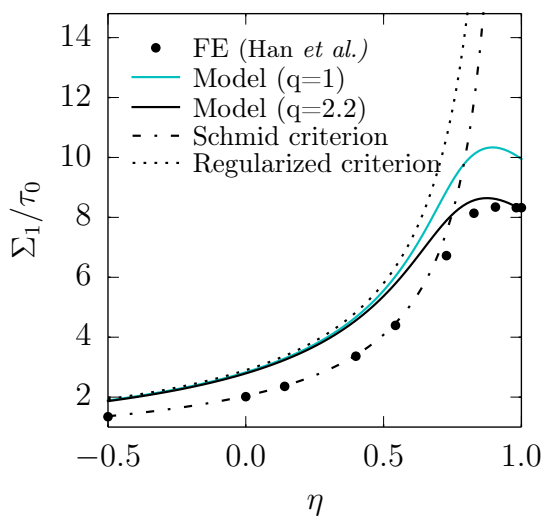

(b) [210] loading direction

Figure 4: Normalized yield stress $\Sigma_{1} / \tau_{0}$ as a function of the axisymmetric loading parameter $\eta=\eta_{2}=\eta_{3}$ for porous FCC single crystals. The yield criterion using a quadratic regularized Schmid law (26) (solid lines) is compared to unit-cell computations reported in [28] (points). Porosity $f=1 \%$. The yield stress of the bulk single crystal according to the standard and quadratic regularized Schmid law is indicated (dashed and dotted lines).

\subsection{Numerical assessment of the generalized criterion}

Following the disproof of the quadratic criterion, attention is now paid to the detailed assessment of the generalized criterion (39). In the absence of porosity, this criterion is arbitrarily close to the Schmid yield surface by considering $n \rightarrow+\infty$. In practice, it has been checked that $n=100$ leads to a correct description. The parameter $q$ has been fitted for a fixed porosity $f=1 \%$ by considering various crystalline orientations and macroscopic loadings (axisymmetric and non-axisymmetric). Use has been made of the FE results of [28] for 32 different cases (8 crystalline orientations with 4 loading conditions) and a correct agreement has been obtained with $q=1.59$. It is pointed out that the value of this correcting factor is consistent with previous studies for a porous material with a von 
Mises matrix [38, 40, 41, 42]. The comparisons are reported in Figure 5 for axisymmetric loadings and Figure 6 for non-axisymmetric loadings. For the latter case, representative examples have been chosen for illustration. For the axisymmetric loadings, two different representations of the results are shown : (i) the yield stress $\Sigma_{1}$ as a function of the loading parameter $\eta$ and (ii) the von Mises stress $\Sigma_{\text {eq }}=\Sigma_{1}(1-\eta)$ as a function of the mean stress $\Sigma_{m}=\frac{1}{3} \Sigma_{1}(1+2 \eta)$. For the axisymmetric loading cases, a smooth (differentiable) evolution of the yield stress with $\eta$ is obtained. As noted in [28], this is related to the activation of the same slip systems whatever the $\eta$ value. An overall good agreement is observed with a slightly less accurate description of the [111] loading case (Figure 5). For non-axisymmetric loadings, different slip systems are activated depending on the value of the loading parameter $\eta_{3}$ (for a fixed value $\eta_{2}$ ). This leads to discontinuities on the slope of the yield stress as described by our criterion. In this case, a very good match is also obtained with the FE unit-cell computations.

Finally, for a further assessment of the proposed criterion, we have also considered a variation of the porosity. Following [28], three crystalline orientations have been considered with axisymmetric loadings and porosities ranging from $0.5 \%$ to $10 \%$. The comparisons are reported in Figure 7 . As a whole, a good description of the yield stress of the porous crystal is obtained for orientations corresponding to single slip ([125] loading direction) or multiple slip ([100] and [111] loading directions). As with the results for a porosity of 1\%, a slightly higher discrepancy is observed for the [111] loading case.

\section{Concluding remarks}

This work represents a contribution to the study of the ductile failure of crystalline materials. More precisely, it has been focused on the description of the yield function of voided single crystals, a topic as yet little discussed despite its importance. By making use of a regularized form of the Schmid law (yield function of the bulk single crystal), a Gurson-type model is proposed. It is obtained by a phenomenological extension of the Benzerga-Besson criterion [9]. Besides, it makes use of the limit-analysis calculation for a hollow sphere, whose matrix presents a Schmid yield locus, which is subjected to a hydrostatic loading. This criterion has been successfully compared with reference numerical results [28] for FCC crystals. Regarding this overall good agreement, it is worth noting that a single fitting parameter has been introduced in our criterion.

These results offer an attractive framework to further envisage the study of crystals presenting different families of slip systems (hexagonal crystalline structure, for instance) as well as the influence of the shape of the void on the macroscopic yield surface. It is particularly suitable for an implementation in FEM codes for structural computations involving crystalline materials (e.g. single crystal turbine blades). Comparisons between the predictions of the model and micromechanical calculations of void growth [see, for instance, 24] will be determinant in order to assess the complete constitutive model. Efforts are currently underway to tackle these questions.

\section{Appendix A. Limit-analysis for a hollow sphere made of an orthotropic Hill matrix}

The main steps of the derivation of the yield criterion (26) are recalled. For further details, the reader is referred to $[9,10]$.

Following [1], the trial velocity field adopted in [9] is of the form:

$$
\mathbf{v}=\frac{b^{3}}{r^{2}} D_{m} \mathbf{e}_{r}+\mathbf{D}^{\prime} \cdot \mathbf{x}
$$



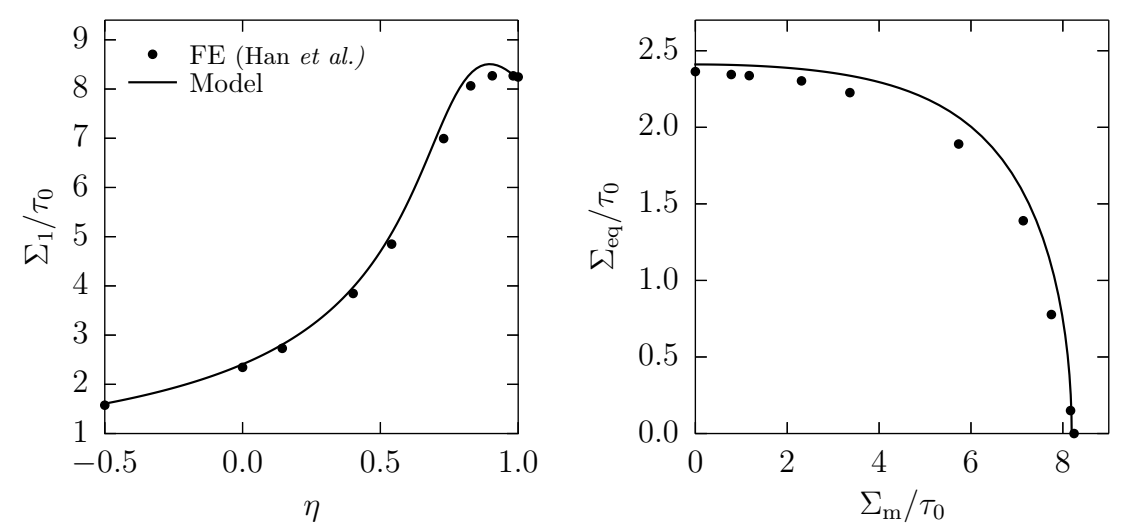

(a) [100] loading direction
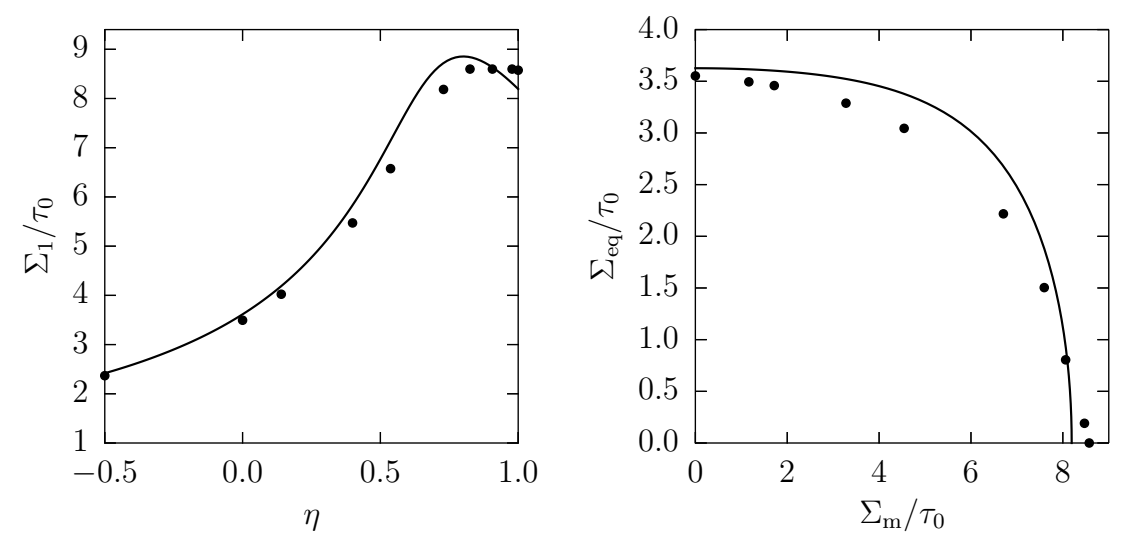

(b) [111] loading direction
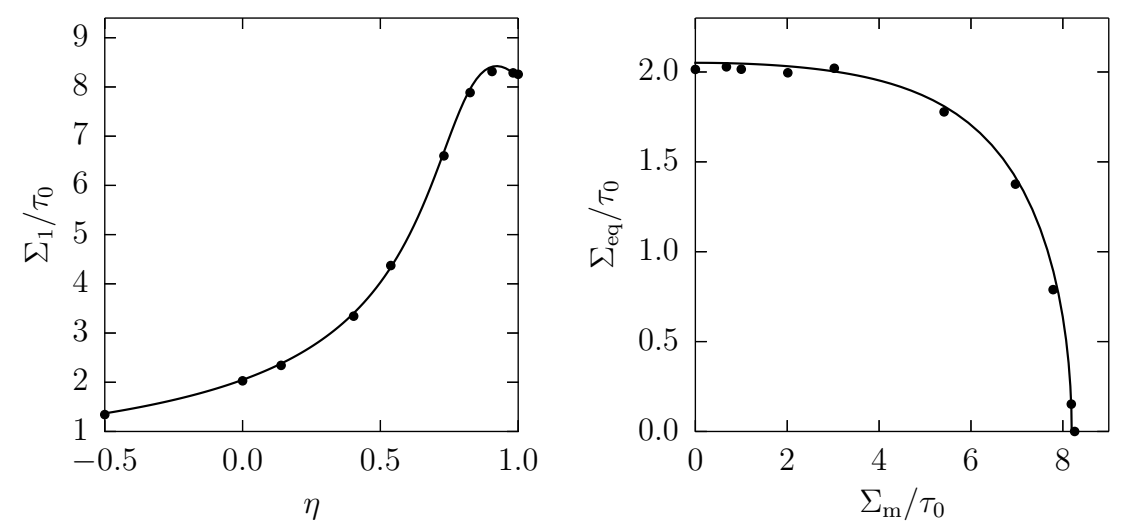

(c) [210] loading direction

Figure 5: Normalized yield stress $\Sigma_{1} / \tau_{0}$ as a function of the axisymmetric loading parameter $\eta=\eta_{2}=\eta_{3}$ (Left) and correponding yield surface in the $\left(\Sigma_{\text {eq }}, \Sigma_{\mathrm{m}}\right)$ plane (Right) for porous FCC single crystals. The yield criterion using a regularized Schmid law with $n=100$ (solid lines) is compared to unit-cell computations reported in [28] (points). Porosity $f=1 \%$. 


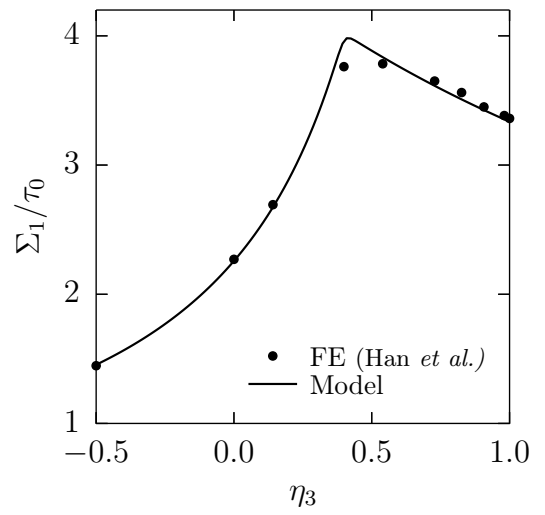

(a) $[100][02 \overline{1}][012]$

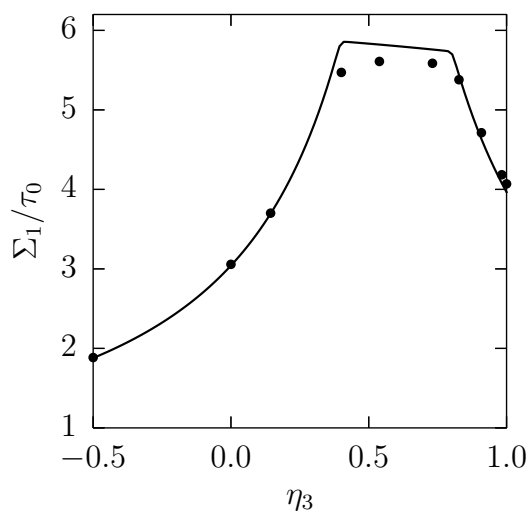

(c) $[111][\overline{2} 11][0 \overline{1} 1]$

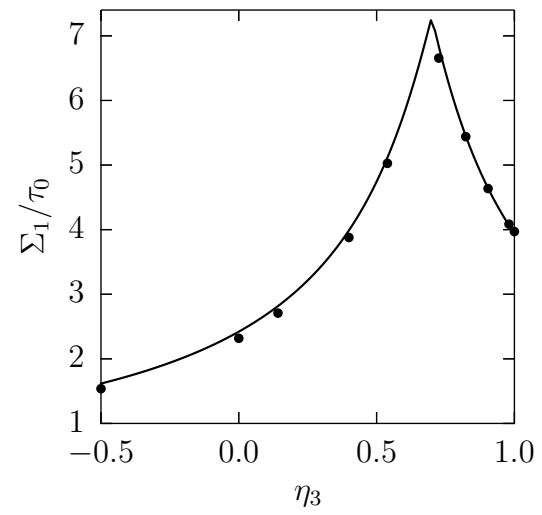

(b) $[110][\overline{1} 10][001]$

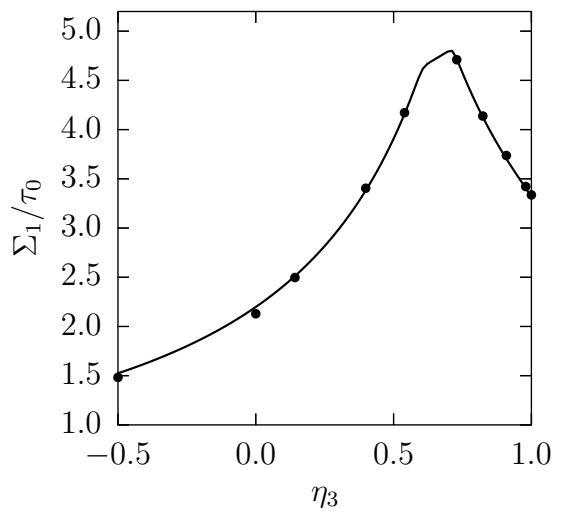

(d) $[1 \overline{2} 5][052][\overline{29} \overline{2} 5]$

Figure 6: Normalized yield stress $\Sigma_{1} / \tau_{0}$ as a function of the loading parameter $\eta_{3}$ with fixed value $\eta_{2}=0.4$ for porous FCC single crystals with different orientations. The yield criterion using a regularized Schmid law with $n=100$ (solid lines) is compared to unit-cell computations reported in [28] (points). Porosity $f=1 \%$. 

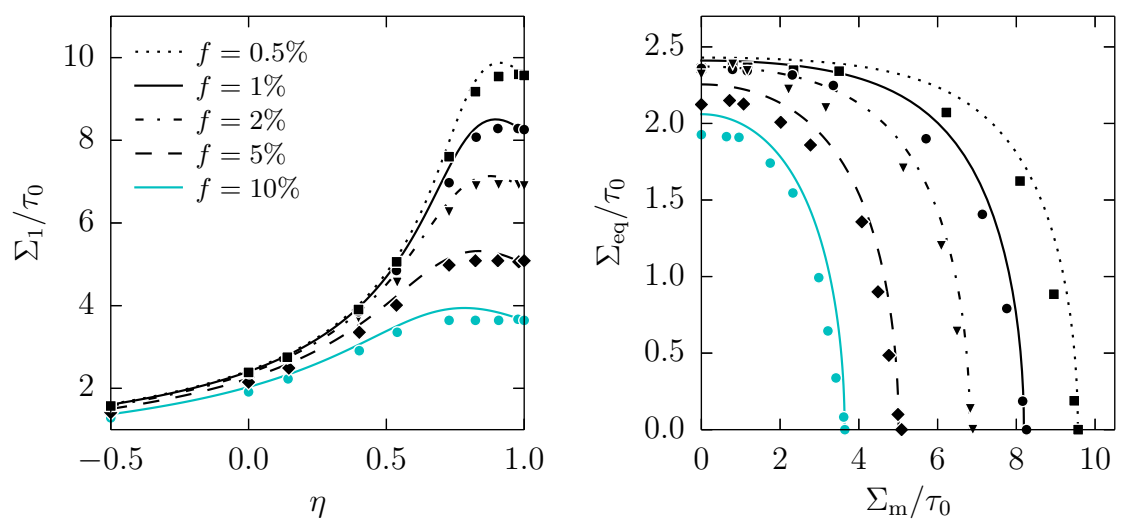

(a) [100] loading direction
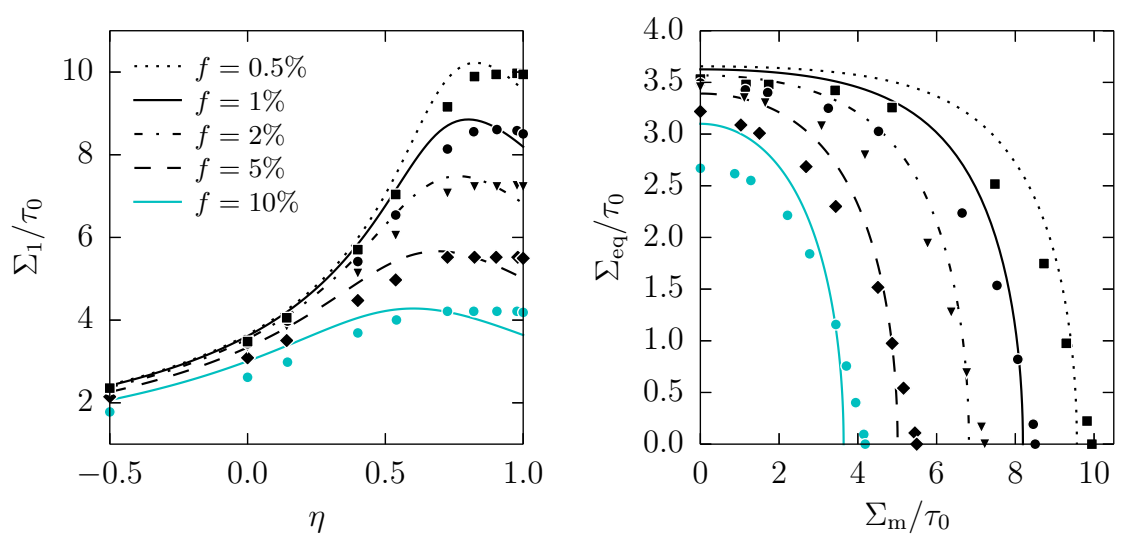

(b) [111] loading direction
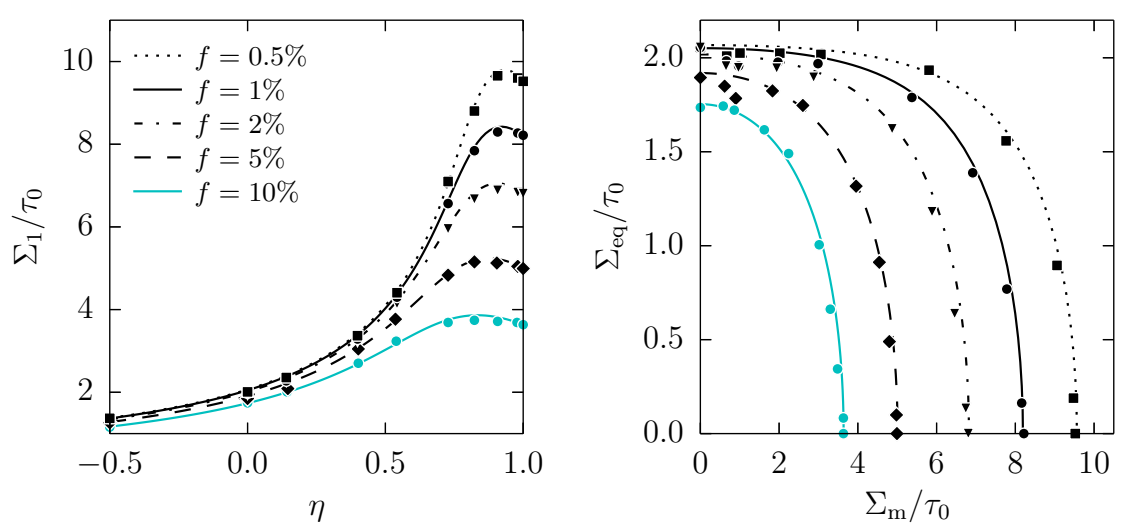

(c) [125] loading direction

Figure 7: Normalized yield stresses $\Sigma_{1} / \tau_{0}$ as a function of the axisymmetric loading parameter $\eta=\eta_{2}=\eta_{3}$ (Left) and correponding yield surfaces in the $\left(\Sigma_{\mathrm{eq}}, \Sigma_{\mathrm{m}}\right)$ plane (Right) for porous FCC single crystals with different loading directions and porosities ranging from $0.5 \%$ to $10 \%$. The yield criterion using a regularized Schmid law with $n=100$ (lines) is compared to unit-cell computations reported in [28] (points). 
where $D_{m}=\frac{1}{3} \operatorname{tr}(\mathbf{D})$ is the macroscopic mean strain rate and $\mathbf{D}^{\prime}$ is the macroscopic deviatoric strain rate tensor. The local strain rate tensor can be decomposed into $\mathbf{d}=\mathbf{d}^{A}+\mathbf{d}^{B}$ with

$$
\mathbf{d}^{A}=\frac{b^{3}}{r^{3}} D_{m}\left(-2 \mathbf{e}_{r} \otimes \mathbf{e}_{r}+\mathbf{e}_{\theta} \otimes \mathbf{e}_{\theta}+\mathbf{e}_{\varphi} \otimes \mathbf{e}_{\varphi}\right) \quad \text { and } \quad \mathbf{d}^{B}=\mathbf{D}^{\prime} .
$$

The local plastic dissipation is thus given by

$$
\pi(\mathbf{d})=\tau_{0} \sqrt{\mathbf{d}^{A}: \mathbf{H}: \mathbf{d}^{A}+\mathbf{d}^{B}: \mathbf{H}: \mathbf{d}^{B}+2 \mathbf{d}^{A}: \mathbf{H}: \mathbf{d}^{B}} .
$$

The macroscopic plastic dissipation defined by

$$
\Pi(\mathbf{D})=\frac{1}{\operatorname{vol}(\Omega)} \int_{\Omega} \pi(\mathbf{d}) \mathrm{d} \Omega
$$

is rewritten, after some approximations, under the form

$$
\Pi(\mathbf{D})=\frac{\tau_{0}}{b^{3}} \int_{\xi}^{\xi f} \sqrt{1+u^{2}} \frac{\mathrm{d} u}{u^{2}} \quad \text { with } \quad \xi \equiv \frac{3}{\kappa} \frac{D_{m}}{D_{e q}} .
$$

$D_{e q}=\sqrt{\mathbf{D}: \mathbf{H}: \mathbf{D}}$ is the macroscopic equivalent strain rate and $\kappa$ is an anisotropy parameter defined by

$$
\kappa=\frac{3}{2} \sqrt{\frac{5}{2 h_{1}+h_{2}+2 h_{3}}} .
$$

The coefficients $\left(h_{1}, h_{2}, h_{3}\right)$ are related to the components of Hill's anisotropic tensor $\mathbf{H}$ as follows

$$
h_{1}=\frac{3}{8}\left(2 H_{11}+2 H_{22}+2 H_{66}-H_{33}\right), \quad h_{2}=\frac{9}{4} H_{33} \quad \text { and } \quad h_{3}=\frac{3}{4}\left(H_{44}+H_{55}\right)
$$

where use has been made of the Kelvin notation [43]. Finally, the definition (15) leads to the expression of the approximate quadratic yield criterion (26). For cubic symmetry, we have $H_{11}=H_{22}=H_{33}$ and $H_{44}=H_{55}=H_{66}$. Besides, by using the cubic spectral decomposition (22), the coefficients can alternatively be expressed as

$$
h_{1}=\frac{3}{4}\left(\frac{1}{\alpha^{\prime}}+\frac{1}{\alpha^{\prime \prime}}\right), \quad h_{2}=\frac{3}{2 \alpha^{\prime}} \quad \text { and } \quad h_{3}=\frac{3}{2 \alpha^{\prime \prime}} .
$$

In the isotropic case, that is $\mathbf{M}=\frac{3}{2} \mathbf{K}\left(\alpha^{\prime}=\alpha^{\prime \prime}=\frac{3}{2}\right)$, the coefficients $h_{1}=h_{2}=h_{3}=1$ and the criterion reduces to the Gurson model [1].

\section{Appendix B. Comparison of the criterion (39) with Han et al. yield function [28]}

Han et al. [28] have derived a multi-criterion yield function for porous FCC single crystals from the variational homogenization method. Inspired by limit-analysis results, the authors have proposed its generalization into a phenomenological yield function. Han et al. criterion reads

$$
\sup _{k=1, \ldots, K} \tau_{k}^{*}-\tau_{0}=0
$$

with $\tau_{k}^{*}$ an effective resolved stress on slip system $k$ defined by

$$
\left(\frac{\boldsymbol{\mu}_{k}: \boldsymbol{\Sigma}}{\tau_{k}^{*}}\right)^{2}+\alpha f \frac{2}{45}\left(\frac{\Sigma_{\mathrm{eq}}}{\tau_{k}^{*}}\right)^{2}+2 q_{1} f \cosh \left(q_{2} \sqrt{\frac{3}{20}} \frac{\Sigma_{m}}{\tau_{k}^{*}}\right)-1-\left(q_{1} f\right)^{2}=0
$$

where $\alpha, q_{1}$ and $q_{2}$ are parameters which needs to be adjusted. It is thus closely related to the yield function that we propose although obtained by a different approach. Our criterion (39) can be seen as a regularized version of a particular case of Han's criterion $(\alpha=0)$. The following differences between the criterions are worth mentioning: 
- Han et al. derived a multi-criterion yield function whereas we propose a single yield function.

- The Han et al. criterion presents a dependence on the equivalent macroscopic stress.

- The Han et al. proposal involves an additional adjusted parameter $\alpha$. It is thus expected to lead, in general, to a better agreeement with unit-cell computations.

\section{Appendix C. Crystalline orientation and rotation matrix}

Throughout the article, the orientation of the single crystal is described by using the so-called Miller indices notation. This description, which is equivalent to an inverse pole figure representation, expresses the unit vectors of the macroscopic frame in the crystalline frame.

Let $\left(\mathbf{e}_{1}, \mathbf{e}_{2}, \mathbf{e}_{3}\right)$ an orthonormal basis of the macroscopic reference frame and $\left(\mathbf{e}_{1}^{\prime}, \mathbf{e}_{2}^{\prime}, \mathbf{e}_{3}^{\prime}\right)$ an orthonormal basis of the crystalline frame. A crystalline orientation denoted $[u v w]-[m n p]-[h k l]$ thus corresponds to

$$
\left\{\begin{array}{l}
\mathbf{e}_{1}=\frac{1}{\sqrt{u^{2}+v^{2}+w^{2}}}\left(u \mathbf{e}_{1}^{\prime}+v \mathbf{e}_{2}^{\prime}+w \mathbf{e}_{3}^{\prime}\right) \\
\mathbf{e}_{2}=\frac{1}{\sqrt{m^{2}+n^{2}+p^{2}}}\left(m \mathbf{e}_{1}^{\prime}+n \mathbf{e}_{2}^{\prime}+p \mathbf{e}_{3}^{\prime}\right) \\
\mathbf{e}_{3}=\frac{1}{\sqrt{h^{2}+k^{2}+l^{2}}}\left(h \mathbf{e}_{1}^{\prime}+k \mathbf{e}_{2}^{\prime}+l \mathbf{e}_{3}^{\prime}\right)
\end{array}\right.
$$

and its orientation matrix $P$ reads

$$
P=\left(\begin{array}{ccc}
\frac{u}{\sqrt{u^{2}+v^{2}+w^{2}}} & \frac{m}{\sqrt{m^{2}+n^{2}+p^{2}}} & \frac{h}{\sqrt{h^{2}+k^{2}+l^{2}}} \\
\frac{v}{\sqrt{u^{2}+v^{2}+w^{2}}} & \frac{n}{\sqrt{m^{2}+n^{2}+p^{2}}} & \frac{k}{\sqrt{h^{2}+k^{2}+l^{2}}} \\
\frac{w}{\sqrt{u^{2}+v^{2}+w^{2}}} & \frac{p}{\sqrt{m^{2}+n^{2}+p^{2}}} & \frac{l}{\sqrt{h^{2}+k^{2}+l^{2}}}
\end{array}\right) .
$$

Let $T_{i j}$ the components of a second order tensor $\mathbf{T}$ in the macroscopic refence frame and $T_{k l}^{\prime}$ its components in the crystalline reference frame. They are related by

$$
T_{i j}=P_{k i} P_{l j} T_{k l}^{\prime} \quad \text { and } \quad T_{k l}^{\prime}=P_{k i} P_{l j} T_{i j}
$$

\section{References}

[1] A. Gurson, Continuum theory of ductile rupture by void nucleation and growth: Part I - Yield criteria and flow rules for porous ductile media., J. Eng. Mater. Technol. 99 (1977) 1-15.

[2] J. Mandel, Contribution théorique à l'étude de l'écrouissage et des lois de l'écoulement plastique, in: Applied Mechanics, Springer, 1966, pp. 502-509.

[3] R. Hill, The essential structure of constitutive laws for metal composites and polycrystals, J. Mech. Phys. Solids 15 (1967) 79-95.

[4] M. Gologanu, J.-B. Leblond, J. Devaux, Approximate models for ductile metals containing nonspherical voids-Case of axisymmetric prolate ellipsoidal cavities, J. Mech. Phys. Solids 41 (1993) $1723-1754$. 
[5] M. Gologanu, J.-B. Leblond, J. Devaux, Approximate models for ductile metals containing nonspherical voids-Case of axisymmetric oblate ellipsoidal cavities, J. Eng. Mat. Tech. 116 (1994) 290-297.

[6] M. Gologanu, J.-B. Leblond, G. Perrin, J. Devaux, Recent extensions of Gurson's model for porous ductile metals, in: P. Suquet (Ed.), Continuum Micromechanics, Springer-Verlag, NewYork, 1997, pp. 61-130.

[7] K. Madou, J.-B. Leblond, A Gurson-type criterion for porous ductile solids containing arbitrary ellipsoidal voids-I: Limit-analysis of some representative cell, J. Mech. Phys. Solids 60 (2012) $1020-1036$.

[8] K. Madou, J.-B. Leblond, A Gurson-type criterion for porous ductile solids containing arbitrary ellipsoidal voids-II: Determination of yield criterion parameters, Journal of the Mechanics and Physics of Solids 60 (5) (2012) 1037-1058.

[9] A. A. Benzerga, J. Besson, Plastic potentials for anisotropic porous solids, Eur. J. Mech. A/Solids 20 (2001) 397-434.

[10] V. Monchiet, O. Cazacu, E. Charkaluk, D. Kondo, Macroscopic yield criteria for plastic anisotropic materials containing spheroidal voids, Int. J. Plast. 24 (2008) 1158-1189.

[11] S. M. Keralavarma, A. A. Benzerga, A constitutive model for plastically anisotropic solids with non-spherical voids, J. Mech. Phys. Solids 58 (2010) 874-901.

[12] F. Pastor, J. Pastor, D. Kondo, Limit analysis of hollow spheres or spheroids with hill orthotropic matrix, C. R. Mecanique 340 (2012) 120-129.

[13] L. Morin, K. Madou, J.-B. Leblond, D. Kondo, A new technique for finite element limit-analysis of hill materials, with an application to the assessment of criteria for anisotropic plastic porous solids, Int. J. Engng Sci. 74 (2014) 65-79.

[14] P. Ponte Castañeda, The effective mechanical properties of nonlinear isotropic composites, J. Mech. Phys. Solids 39 (1991) 45-71.

[15] P. Ponte Castañeda, P. Suquet, Nonlinear composites, Adv. Appl. Mech. 34 (1998) 171-302.

[16] P. Ponte Castañeda, Second-order homogenization estimates for nonlinear composites incorporating field fluctuations. I - Theory, J. Mech. Phys. Solids 50 (2002) 737-757.

[17] M. I. Idiart, P. Ponte Castañeda, Variational linear comparison bounds for nonlinear composites with anisotropic phases. I. General results, Proc. R. Soc. Lond. A463 (2007) 907-924.

[18] R. A. Lebensohn, M. I. Idiart, P. Ponte Castañeda, P.-G. Vincent, Dilatational viscoplasticity of polycrystalline solids with intergranular cavities, Phil. Mag. 91 (2011) 3038-3067.

[19] M. I. Idiart, J. E. R. Nervi, Bounds on the hydrostatic plastic strength of voided polycrystals and implications for linear-comparison homogenization techniques, C. R. Mecanique 342 (2014) $25-31$.

[20] R. A. Lebensohn, J. P. Escobedo, E. K. Cerreta, D. Dennis-Koller, C. A. Bronkhorst, J. F. Bingert, Modeling void growth in polycrystalline materials, Acta Mater. 61 (2013) 6918-6932. 
[21] J. W. Kysar, Y. X. Gan, G. Mendez-Arzuza, Cylindrical void in a rigid-ideally plastic single crystal. Part I: Anisotropic slip line theory solution for face-centered cubic crystals, Int. J. Plast. 21 (2005) 1481-1520.

[22] Y. X. Gan, W. Kysar, T. L. Morse, Cylindrical void in a rigid-ideally plastic single crystal II: Experiments and simulations, Int. J. Plast. 22 (2006) 39-72.

[23] T. Schacht, N. Untermann, E. Steck, The influenceof crystallographic orientation on the deformation behaviour of single crystals containing microvoids, Int. J. Plast. 19 (2003) 1605-1626.

[24] S. Yerra, C. Tekoglu, F. Scheyvaerts, L. Delannay, P. V. Houtte, T. Pardoen, Void growth and coalescence in single crystals, Int. J. Solids Struct. 47 (2010) 1016-1029.

[25] A. Srivastava, A. Needleman, Void growth versus void collapse in a creeping single crystal, J. Mech. Phys. Solids 61 (2013) 1169-1184.

[26] M. I. Idiart, P. Ponte Castañeda, Variational linear comparison bounds for nonlinear composites with anisotropic phases. II. crystalline materials, Proc. R. Soc. Lond. A463 (2007) 925-943.

[27] G. de Botton, P. Ponte Castañeda, Variational estimates for the creep behaviour of polycrystals, Proc. R. Soc. Lond. A448 (1995) 121-142.

[28] X. Han, J. Besson, S. Forest, B. Tanguy, S. Bugat, A yield function for single crystals containing voids, Int. J. Solids Struct. 50 (2013) 2115-2131.

[29] M. Arminjon, A regular form of the schmid law. Application to the ambiguity problem., Texture Microstruct. 14-18 (1991) 1121-1128.

[30] W. Gambin, Refined analysis of elastic-plastic crystals, Int. J. Solids Struct. 29 (1992) 2013-2021.

[31] E. Schmid, W. Boas, Kristallplastizität, Springer, 1935.

[32] F. Montheillet, P. Gilormini, J. J. Jonas, Relation between axial stresses and texture development during torsion testing: a simplified theory, Acta Metall. 33 (1985) 705-717.

[33] M. Darrieulat, D. Piot, A method of generating analytical yield surfaces of crystalline materials, Int. J. Plasticity 12 (1996) 575-610.

[34] J. F. W. Bishop, R. Hill, A theoretical derivation of the plastic properties of a polycrystalline face-centred metal, Phil. Mag. 42 (1951) 1298-1307.

[35] P. Suquet, Elements of homogenization for inelastic solid mechanics, in: E. Sanchez-Palencia, A. Zaoui (Eds.), Homogenization Techniques for Composite Media, Springer-Verlag, 1987, pp. 194-278.

[36] R. Hill, A theory of the yielding and plastic flow of anisotropic metals, Proc. R. Soc. Lond. A193 (1948) 281-297.

[37] L. J. Walpole, Elastic behavior of composite materials: theoretical foundations, Adv. Appl. Mech. 21 (1981) 169-242.

[38] V. Tvergaard, On localization in ductile materials containing spherical voids, Int. J. Fract. 18 (1982) 237-252. 
[39] P.-G. Vincent, P. Suquet, Y. Monerie, H. Moulinec, Effective flow surface of porous materials with two populations of voids under internal pressure: II. Full-field simulations, Int. J. Plast. 56 (2014) 74-98.

[40] G. Perrin, J. B. Leblond, Analytical study of a hollow sphere made of plastic porous material and subjected to hydrostatic tension - Application to some problems in ductile fracture of metals, Int. J. Plast. 6 (1990) 677-699.

[41] J.-B. Leblond, G. Perrin, P. Suquet, Exact results and approximate models for porous viscoplastic solids, Int. J. Plast. 10 (1994) 213-235.

[42] F. Fritzen, S. Forest, T. Böhlke, D. Kondo, T. Kanit, Computational homogenization of elastoplastic porous metals, Int. J. Plast. 29 (2012) 102-119.

[43] M. M. Mehrabadi, S. C. Cowin, Eigentensors of linear anisotropic elastic materials, Quart. J. Mech. Appl. Math. 43 (1990) 15-41. 